\title{
QUASIAFFINE TRANSFORMS OF OPERATORS
}

\author{
C. Apostol, H. Bercovici, C. Foiaş and C. Pearcy
}

1. Introduction. Let $\mathfrak{H}$ and $\mathfrak{H}^{\prime}$ be separable, infinite dimensional, complex Hilbert spaces, and let $\mathscr{L}\left(\mathcal{H C}^{\prime}, \mathcal{H}\right)$ denote the space of all bounded, linear operators from $\mathcal{H C}^{\prime}$ to $\mathcal{H}$. If $\mathfrak{H C}^{\prime}=\mathcal{H}$, we write $\mathscr{L}(\mathcal{H C})$ in place of $\mathscr{L}\left(\mathcal{H C}^{\prime}, \mathfrak{H}\right)$. An $X$ in $\mathscr{L}\left(\mathfrak{H}^{\prime}, \mathfrak{H C}\right)$ is called a quasiaffinity if it has trivial kernel and dense range. An operator $T^{\prime}$ in $\mathcal{L}\left(\mathcal{F}^{\prime}\right)$ is said to be a quasiaffine transform of an operator $T$ in $\mathscr{L}(\mathfrak{H C})$ (notation: $\left.T^{\prime} \prec T\right)$ if there exists a quasiaffinity $X$ in $\mathscr{L}\left(\mathcal{H C}^{\prime}, \mathfrak{H C}\right)$ such that $X T^{\prime}=T X$. If both $T^{\prime}<T$ and $T<T^{\prime}$, then we say that $T^{\prime}$ and $T$ are quasisimilar, and we write $T^{\prime} \sim T$. These relations have already played a considerable role in operator theory (see, for example, [13]), and under certain additional hypotheses, one knows that $T^{\prime}<T$ implies $T^{\prime} \sim T$. This is true when $T$ and $T^{\prime}$ are normal [13], and also when $T$ and $T^{\prime}$ belong to the class $C_{0}$ in the terminology of [13], cf. [17]. Moreover, if $T^{\prime}<T$ and $T$ is algebraic, i.e., satisfies some polynomial equation, then $T^{\prime}$ is also algebraic and $T^{\prime} \sim T$ [2], [19]. However, it has been known for a long time (cf. [12] and [16]) that the relation " $\prec$ " is not reflexive, and that, in general, one may have $T^{\prime} \prec T$ without $T^{\prime}$ inheriting many of the properties of $T$. For nonalgebraic operators this phenomenon is quite striking. Indeed, it was shown in [16] that if $T$ is a nonalgebraic strict contraction having a cyclic vector, then the unilateral unweighted shift $S^{(\infty)}$ of infinite multiplicity is a quasiaffine transform of $T$. We will show in Section 3 that this result remains true if the hypothesis of possessing a cyclic vector is omitted. It will follow that every nonalgebraic operator in $\mathscr{L}(\mathcal{J C})$ has a quasiaffine transform of the form $\alpha S^{(\infty)}$ for some positive number $\alpha$.

In Section 4 we will use the main theorem of Section 3 and a result of Berg [4] to prove that every $T$ in $\mathcal{L}(\mathcal{J C})$ has a quasiaffine transform of the form $N+K$ where $N$ is normal and $K$ is a compact operator of arbitrarily small norm. Of course, as noted above, if $T$ is algebraic, then $T$ and $N+K$ are quasisimilar. Unfortunately the relation $N+K \prec T$ does not always imply quasisimilarity. Indeed, we show that a quasinilpotent operator which is quasisimilar to an operator of the form $N+K$ (with $N$ normal and $K$ compact) necessarily commutes with a nonzero compact operator, and one knows from [7] that there are quasinilpotent operators that do not commute with any nonzero compact operator.

Finally, we improve a result from [7] by showing that any operator $T$ in $\mathscr{L}(\mathcal{H C})$ whose essential spectrum $\sigma_{e}(T)$ is the singleton $\{0\}$ has a compact quasiaffine transform $K$. Again in this case the relation $K<T$ cannot be replaced by quasisimilarity.

We remark that the results in this paper were obtained in 1976 and 1977. Earlier versions of the paper appeared as INCREST preprints in those years, and the main results were announced in the Notices of the A.M.S. in 1978 (78T-B110).

2. Preliminaries. We will denote by $\mathbf{K}=\mathbf{K}(\mathfrak{H C})$ the ideal of compact operators in $\mathscr{L}(\mathfrak{K C})$ and by $\pi: \mathfrak{L}(\mathfrak{H C}) \rightarrow \mathscr{L}(\mathfrak{H C}) / \mathbf{K}$ the canonical projection onto the Calkin

Received April 24, 1981. Revision received June 22, 1981.

Michigan Math. J. 29 (1982). 
algebra. If $T \in \mathcal{L}(\mathcal{H C})$, we denote by $\sigma(T)$ the spectrum of $T$ and by $\sigma_{e}(T)$ the essential spectrum of $T$, i.e., the spectrum of $\pi(T)$. If $n$ is any positive integer, $T^{(n)}$ will denote the direct sum of $n$ copies of $T$ (acting on the direct sum $\mathcal{H C}^{(n)}$ of $n$ copies of $\mathcal{H C}$ ). In this same vein, $T^{(\infty)}$ will denote the direct sum of $\aleph_{0}$ copies of $T$ acting on the Hilbert space $\mathcal{H C}^{(\infty)}$ that is the direct sum of $\aleph_{0}$ copies of $\mathcal{H}$.

We write $D$ for the open unit disc in $\mathbf{C}$ and $H^{2}$ for the Hardy space consisting of those analytic functions on $D$ with square integrable boundary values. Similarly, we denote by $H^{\infty}$ the Banach algebra of all bounded analytic functions on $D$ under the supremum norm. Furthermore, we denote by $S$ the unilateral shift acting on $H^{2}$ defined by

$$
(S u)(\zeta)=\zeta u(\zeta), \quad \zeta \in D, \quad u \in H^{2}
$$

Note that for the canonical basis $\left\{e_{n}\right\}_{n=0}^{\infty}$ of $H^{2}$ defined by $e_{n}(\zeta)=\zeta^{n}$, we have $S e_{n}=e_{n+1}$. More generally, if $\omega=\left\{w_{n}\right\}_{n=0}^{\infty}$ is any bounded sequence of complex numbers, the unilateral weighted shift $W_{\omega}$ is defined on $H^{2}$ by $W_{\omega} e_{n}=w_{n} e_{n+1}$ for $n \geqslant 0$.

Observe next that if $T$ is a strict contraction (i.e., $\|T\|<1$ ) in $\mathscr{L}\left(\mathcal{H}\right.$ ) and $\phi \in H^{2}$, then $\phi$ is analytic in a neighborhood of $\sigma(T)$ and therefore we can define the operator $\phi(T)$ by the Riesz-Dunford functional calculus. For a fixed $h$ in $\mathcal{H C}$ (and fixed $T$ ) we denote by $X_{h}: H^{2} \rightarrow \mathcal{H C}$ the operator defined by $X_{h}(\phi)=\phi(T) h$ for all $\phi$ in $H^{2}$. An easy calculation shows that $T X_{h}=X_{h} S$ for all $h$ in $\mathcal{H}$, and the following lemma gives more information about $X_{h}$.

LEMMA 2.1. If $T$ is a fixed strict contraction in $\mathcal{L}(\mathfrak{H})$, then for each $h$ in $\mathcal{H C}$, the operator $X_{h}: H^{2} \rightarrow \mathcal{H C}$ defined above is compact and satisfies $\left\|X_{h}\right\| \leqslant C\|h\|$ where $C$ is a constant depending only on $\|T\|$.

Proof. Let $h$ be a fixed vector in $\mathcal{H}$, let $r$ satisfy $\|T\|<r<1$, and observe that $X_{h}$ is the composition $X_{h}=\Psi_{h} \circ \Phi_{r} \circ Y_{r}$, where $Y_{r}: H^{2} \rightarrow H^{\infty}, \Phi_{r}: H^{\infty} \rightarrow \mathcal{L}(\mathcal{H})$, and $\Psi_{h}: \mathcal{L}(\mathcal{H C}) \rightarrow \mathcal{H C}$ are defined by

$$
\begin{aligned}
\left(Y_{r} \phi\right)(\zeta) & =\phi(r \zeta), \quad \phi \in H^{2}, \zeta \in D \\
\Phi_{r}(\phi) & =\phi\left(r^{-1} T\right), \quad \phi \in H^{\infty} \\
\Psi_{h}(R) & =R h, \quad R \in \mathcal{L}(\mathcal{H C}) .
\end{aligned}
$$

It is easy to see that $Y_{r}, \Phi_{r}$, and $\Psi_{h}$ are norm-continuous and that $\left\|Y_{r}\right\| \leqslant\left(1-r^{2}\right)^{-1 / 2}$, $\left\|\Phi_{r}\right\| \leqslant 1$, and $\left\|\Psi_{h}\right\| \leqslant\|h\|$, from which the inequality $\left\|X_{h}\right\| \leqslant C\|h\|$ follows, where $C$ depends only on $r$ and thus only on $\|T\|$. To show that $X_{h}$ is compact, it suffices to show that $Y_{r}$ is a compact operator. But we can write $Y_{r}=Y_{\sqrt{r}} Z_{\sqrt{r}}$ where $Z_{\sqrt{r}}: H^{2} \rightarrow H^{2}$ is defined by $\left(Z_{\sqrt{r}} \phi\right)(\zeta)=\phi(\sqrt{r} \zeta)$, and the compactness of $Z_{\sqrt{r}}$ is an immediate consequence of the fact that the matrix of $Z_{\sqrt{r}}$ relative to the orthonormal basis $\left\{e_{n}\right\}$ for $H^{2}$ is $\operatorname{Diag}\left(1, r^{1 / 2}, r, r^{3 / 2}, \ldots\right)$.

An operator $T$ in $\mathscr{L}(\mathcal{H})$ is said to be polynomially compact if $p(T) \in \mathbf{K}$ for some nonzero polynomial $p$. It is an easy consequence of the spectral mapping theorem that if $T$ is polynomially compact, then $\sigma_{e}(T)$ is a finite set and $\sigma(T)$ is at most a countable set whose only accumulation points belong to $\sigma_{e}(T)$ (cf. [10, Cor. 1.26]). Furthermore, one knows (cf. [10, Prop. 1.27]) that every $\lambda$ in $\sigma(T) \backslash \sigma_{e}(T)$ is an eigenvalue of $T$ of finite multiplicity. 
LEMMA 2.2. If $T$ is a polynomially compact contraction in $\mathcal{L}(\mathcal{H})$ such that $1-T T^{*}$ has finite rank, then $\sigma_{e}(T)$ is a subset of the unit circle.

Proof. The hypothesis implies that $\pi(T)$ is a coisometry and if this coisometry were not unitary, then $\sigma_{e}(T)$ would have to contain $D$, contradicting the fact that $\sigma_{e}(T)$ is finite. Thus $\pi(T)$ is unitary and the result follows.

COROLLARY 2.3. Let $T$ be as in Lemma 2.2, and let $0<r<1$. Then there exist disjoint subspaces $\mathfrak{H}_{0}$ and $\mathfrak{H C}_{1}$ of $\mathcal{H}$ such that $\mathfrak{H C}_{0}+\mathcal{H C}_{1}=\mathfrak{H}, \operatorname{dim} \mathfrak{H C}_{0}<\infty, T \mathcal{H}_{i} \subset \mathfrak{H C}_{i}$ for $i=1,2$, and $\sigma\left(T \mid \mathcal{H}_{1}\right) \cap\{z:|z| \leqslant r\}=\varnothing$.

Proof. As noted above, the set $\sigma_{0}=\sigma(T) \cap\{z:|z| \leqslant r\}$ must be finite and consist entirely of eigenvalues of $T$ of finite multiplicity. If $E$ is the spectral idempotent for $T$ corresponding to $\sigma_{0}$, then we may set $\mathcal{H}_{0}=E \mathcal{H C}$ and $\mathcal{H}_{1}=(1-E) \mathcal{H}$, and the result follows from the well-known properties of spectral idempotents.

REMARK 2.4. In connection with Lemma 2.2, we also note that if $\mathscr{T} \subset\left(H^{2}\right)^{(n)}$ is an invariant subspace of $S^{(n)}$, and if $T$ is defined by $T=P_{\mathfrak{N}} \perp S^{(n)} \mid \mathfrak{T}^{\perp}$, where $P_{\mathfrak{M}} \perp$ denotes the orthogonal projection onto $\mathfrak{T}^{\perp}$, then

$$
\begin{aligned}
\operatorname{rank}\left(1_{\mathfrak{M}^{\perp}}-T T^{*}\right) & =\operatorname{rank}\left(P_{\mathfrak{M}^{\perp}}\left(1-S^{(n)} S^{(n)^{*}}\right) \mid \mathfrak{T}^{\perp}\right) \\
& \leqslant \operatorname{rank}\left(1-S^{(n)} S^{(n)^{*}}\right)=n,
\end{aligned}
$$

so that $T$ satisfies the conditions of Lemma 2.2 if it is polynomially compact.

In what follows we shall also have occasion to use a certain result on truncated weighted shifts. Let $\omega=\left\{w_{j}\right\}_{j=0}^{n}$ be a finite sequence of complex numbers, and let $T_{\omega}$ be the truncated weighted shift with weight sequence $\omega$ defined on an $(n+2)$ dimensional Hilbert space by setting

$$
T_{\omega} f_{j}=w_{j} f_{j+1}, \quad 0 \leqslant j \leqslant n, \quad \text { and } T_{\omega} f_{n+1}=0,
$$

where $\left\{f_{j}\right\}_{j=0}^{n+1}$ is an orthonormal basis for the space. The following theorem is a slight modification of ([4], Theorem 1).

THEOREM 2.5 (D. Berg). Let $T_{\omega}$ be the truncated weighted shift with weight sequence $\omega=\left\{w_{j}\right\}_{j=0}^{n}$. Suppose that $1 \leqslant\left\|T_{\omega}\right\| \leqslant 2$ and for some $\epsilon>0$, we have $\left|w_{0}\right| \leqslant \epsilon, \quad\left|w_{n}\right| \leqslant \epsilon$, and $\left|\left(\left|w_{j}\right|-\left|w_{j+1}\right|\right)\right| \leqslant \epsilon$ for $0 \leqslant j<n$. Then there exists a normal operator $N$ such that $\left\|T_{\omega}-N\right\|<200 \epsilon^{1 / 2}$.

A typical sequence of weights satisfying the conditions of Theorem 2.5 is obtained as follows. Let $0<\epsilon<1$, and define the finite sequence $\omega(\epsilon, k)$ to be the weight sequence

$$
\epsilon, 2 \epsilon, 3 \epsilon, \ldots,(n-1) \epsilon, n \epsilon, n \epsilon, \ldots, n \epsilon,(n-1) \epsilon, \ldots, 2 \epsilon, \epsilon
$$

where $n$ is the smallest integer such that $n \epsilon>1$ and the term $n \epsilon$ appears $k$ times. The truncated weighted shift with weight sequence $\omega(\epsilon, k)$ will be denoted by $T_{\omega(\epsilon, k)}$.

In a different direction, recall that an operator $T$ in $\mathscr{L}(\mathcal{H C})$ is quasinilpotent if $\sigma(T)=\{0\}$. The following result is [7, Theorem 1].

THEOREM 2.6. Let $T$ be a quasinilpotent operator in $\mathcal{L}(\mathcal{H C})$. Then there exist a compact quasinilpotent operator $K$ in $\mathscr{L}(\mathcal{H})$ and an operator $X$ in $\mathscr{L}\left(\mathcal{H}^{C}, \mathcal{H}^{(\infty)}\right)$ that is bounded below such that $K^{(\infty)} X=X T$. 
We will also need the following result which follows from [18] or [3].

PROPOSITION 2.7. Any operator $T$ in $\mathscr{L}(\mathcal{H C})$ such that $\sigma_{e}(T)=\{0\}$ can be written as $T=T^{\prime}+K$ where $T^{\prime}$ is quasinilpotent and $K$ is compact.

3. The shift as a universal quasiaffine transform. In this section we will prove that every nonalgebraic operator in $\mathscr{L}(\mathcal{H C})$ has a quasiaffine transform which is a multiple of $S^{(\infty)}$. The following two lemmas are the main steps needed to reduce the proof of this theorem to a more manageable one.

LEMMA 3.1. Let $T$ in $\mathcal{L}(\mathcal{H C})$ and $T^{\prime}$ in $\mathcal{L}\left(\mathcal{F C}^{\prime}\right)$ be two strict contractions such that $S \oplus T^{\prime} \prec T$. Then $\alpha S^{(\infty)} \prec T$ for every scalar $\alpha>1$.

Proof. If $\alpha>1$, by the results of [16] we have $\alpha S^{(\infty)} \prec S$ and hence $\alpha S^{(\infty)} \oplus T^{\prime} \prec$ $S \oplus T^{\prime}$. By the transitivity of the relation " $\prec$ " it is enough to show that $\alpha S^{(\infty)} \prec$ $\alpha S^{(\infty)} \oplus T^{\prime}$. Setting $T^{\prime \prime}=\alpha^{-1} T^{\prime}$, this relation is equivalent to

$$
\bigoplus_{i, j=0}^{\infty} S<\left(\bigoplus_{i, j=0}^{\infty} S\right) \oplus T^{\prime \prime}
$$

Let us choose a sequence $\left\{h_{j}\right\}_{j=0}^{\infty}$, dense in the unit ball of $\mathcal{F C}^{\prime}$, and define the operator $X: \oplus_{i, j=0}^{\infty} H^{2} \rightarrow\left(\oplus_{i, j=0}^{\infty} H^{2}\right) \oplus \mathcal{J C}^{\prime}$ by

$$
X\left(\bigoplus_{i, j=0}^{\infty} \phi_{i j}\right)=\left(\bigoplus_{i, j=0}^{\infty} 4^{-i-j} \phi_{i j}\right) \oplus\left(\sum_{i, j=0}^{\infty} 2^{-i-j} \phi_{i j}\left(T^{\prime \prime}\right) h_{j}\right) .
$$

That $X$ is bounded follows from the fact that $\phi_{i j}\left(T^{\prime \prime}\right) h_{j}=X_{h_{j}}\left(\phi_{i j}\right)$ and Lemma 2.1. It is obvious that $X$ is one-to-one and that $\left(\left(\oplus_{i, j=0}^{\infty} S\right) \oplus T^{\prime \prime}\right) X=X\left(\oplus_{i, j=0}^{\infty} S\right)$. To conclude the proof it therefore suffices to show that $X$ has dense range. It is easy to see that the relation

$$
\left(\bigoplus_{i, j=0}^{\infty} \psi_{i j}\right) \oplus k \in(\text { range } X)^{\perp}
$$

is equivalent to

$$
4^{-i-j}\left(\phi, \psi_{i j}\right)_{H^{2}}+2^{-i-j}\left(\phi\left(T^{\prime \prime}\right) h_{j}, k\right)_{\mathcal{H C}}{ }^{\prime}=0
$$

for all $i, j \geqslant 0$ and $\phi$ in $H^{2}$. Therefore we infer that

$$
\left(\phi\left(T^{\prime \prime}\right) h_{j}, k\right)_{\mathcal{H}}{ }^{\prime}=\lim _{i \rightarrow \infty} 2^{-i-j}\left(\phi, \psi_{i j}\right)=0
$$

and hence $k=0$ because the sequence $\left\{h_{j}\right\}_{j=0}^{\infty}$ is dense. (Note that for $\phi=1$, $\left.\phi\left(T^{\prime \prime}\right) h_{j}=h_{j}\right)$. Now the relations (3.2) reduce to $\left(\phi, \psi_{i j}\right)=0$ for all $\phi$ in $H^{2}$, and this clearly implies $\psi_{i j}=0$. Thus (3.1) implies $\left(\oplus_{i, j=0}^{\infty} \psi_{i j}\right) \oplus k=0$, and the lemma follows.

LEMMA 3.2. Let $T$ in $\mathscr{L}(\mathfrak{H C})$ and $T^{\prime}$ in $\mathcal{L}\left(\mathfrak{H C}^{\prime}\right)$ be two strict contractions and let $X$ be an operator in $\mathcal{L}\left(\mathcal{H}^{\prime}, \mathcal{H}\right)$ with dense range such that $T X=X T^{\prime}$. If there exists an $h$ in $\mathcal{H}$ such that for no nonzero polynomial $p$ does $p(T) h$ belong to the range of $X$, then $S \oplus T^{\prime \prime} \prec T$ for some strict contraction $T^{\prime \prime}$. 
Proof. Replacing $T^{\prime}$ and $X$ by $P_{(\operatorname{ker} X)}{ }^{\perp} T^{\prime} \mid(\operatorname{ker} X)^{\perp}$ and $X \mid(\operatorname{ker} X)^{\perp}$, respectively, we may assume that $X$ is one-to-one. Under this additional assumption we will prove that in fact

$$
S \oplus T^{\prime} \prec T .
$$

The operator $Y: H^{2} \oplus \mathcal{H}^{\prime} \rightarrow \mathcal{H}$ defined by $Y\left(\phi \oplus h^{\prime}\right)=\phi(T) h-X h^{\prime}$ has dense range (because range $Y \supset$ range $X)$ and $T Y=Y\left(S \oplus T^{\prime}\right)$. To complete the proof of (3.3) it suffices to show that $Y$ is one-to-one. Thus suppose that $Y\left(\phi \oplus h^{\prime}\right)=0$, that is,

$$
\phi(T) h=X h^{\prime}
$$

Choose a positive $r$ less than 1 such that $r>\max \left\{\|T\|,\left\|T^{\prime}\right\|\right\}$. If the function $\phi$ is not the zero function, we can write $\phi=p \psi$ where $p$ is a polynomial and $\psi$ is a function in $H^{2}$ having no zeros in the disk $\{z:|z| \leqslant r\}$. Then $\psi(T)$ and $\psi\left(T^{\prime}\right)$ are invertible, and therefore $p(T) h=\psi(T)^{-1} \phi(T) h=\psi(T)^{-1} X h^{\prime}=X \psi\left(T^{\prime}\right)^{-1} h^{\prime} \in X \mathcal{H}^{\prime}$. By the hypothesis we have $p=0$ and hence $\phi=0$. Thus (3.4) reduces to $X h^{\prime}=0$, so that $h^{\prime}=0$ by the injectivity of $X$, and the proof is complete.

The preceding lemma suggests the following definition.

DEFINITION 3.3. A strict contraction $T$ in $\mathscr{L}(\mathcal{H C})$ has property (A) if for every strict contraction $T^{\prime}$, every operator $X$ with dense range such that $T X=X T^{\prime}$, and every $h$ in $\mathcal{H}$, there exists a nonzero polynomial $p$ such that $p(T) h$ belongs to the range of $X$.

An easy consequence of Lemmas 3.1 and 3.2 is the following.

PROPOSITION 3.4. If $T$ is a strict contraction in $\mathcal{L}(\mathcal{H})$ not having property (A), we have $\alpha S^{(\infty)}<T$ for every scalar $\alpha>1$.

In the sequel 'we will use the slightly stronger property (B) defined below.

DEFINITION 3.5. A strict contraction $T$ in $\mathscr{L}(\mathcal{H C})$ has property (B) if for every (not necessarily strict) contraction $T^{\prime}$, every operator $X$ with dense range such that $T X=X T^{\prime}$, and every $h$ in $\mathcal{H C}$, there exists a nonzero polynomial $p$ such that $p(T) h$ belongs to the range of $X$.

Note that if $T$ has property (A) and $1<\alpha<\|T\|^{-1}$, then $\alpha T$ has property (B). Furthermore, it is obvious that any algebraic operator has properties (A) and (B). Our strategy consists of proving that the converse is also true: any operator having property $(\mathrm{B})$ is an algebraic operator.

The following lemmas will help us "globalize" property (B).

LEMMA 3.6. Let $X$ be a complex linear space, $n$ be a positive integer, and $T a$ linear operator on $X$. Assume that for each $x$ in $X$ there exists a nonzero polynomial $r_{x}$ of degree less than or equal to $n$ such that $r_{x}(T) x=0$. Then $T$ is an algebraic operator. 
Proof. For each $x$ in $X$ we denote by $p_{x}$ the generator of the ideal $J_{x}=$ $\{p: p(T) x=0\}$. For every pair $x, y$ in $X$ there exists $z$ in $X$ such that $p_{z}$ is the least common multiple $m$ of $p_{x}$ and $p_{y}$. Indeed, it suffices to write $p_{x}=p^{\prime} p^{\prime \prime}, p_{y}=q^{\prime} q^{\prime \prime}$, such that $p^{\prime}$ and $q^{\prime}$ are relatively prime, $p^{\prime} q^{\prime}=m$, and define $z=p^{\prime \prime}(T) x+q^{\prime \prime}(T) y$. The equality $p_{z}=m$ follows from the relations $p_{p^{\prime \prime}(T) x}=p^{\prime}, p_{q^{\prime \prime}(T) y}=q^{\prime}$, and the fact that $p_{u+v}=p_{u} \cdot p_{v}$ when $p_{u}$ and $p_{v}$ are relatively prime. This argument, combined with the assumption that $p_{x}$ is of degree less than or equal to $n$ for each $x$, shows that the family $\left\{p_{x}\right\}_{x \in X}$ admits a common multiple $p$ for which, obviously, $p(T)=0$.

Recall that a linear manifold $\mathfrak{N}$ of $\mathcal{H C}$ is called paraclosed if it is the range of some operator in $\mathscr{L}\left(\mathfrak{H}^{\prime}, \mathfrak{H}\right)$.

LEMMA 3.7. Suppose $T \in \mathscr{L}(\mathcal{H C})$ and $\mathfrak{N}$ is a paraclosed linear manifold of $\mathcal{H C}$ such that TIK $\subset \mathfrak{N}$. If for every $x$ in $\mathcal{H C}$ there exists a nonzero polynomial $p_{x}$ such that $p_{x}(T) x \in \mathfrak{M}$, then $p(T) \mathfrak{H C} \mathscr{N}$ for some nonzero polynomial $p$.

Proof. We may write $\mathfrak{T}=R \mathcal{H C}^{\prime}$ where $R \in \mathscr{L}\left(\mathfrak{H C}^{\prime}, \mathfrak{H C}\right)$. For every positive integer $N$, let us denote by $s_{N}$ the set of all $x$ in $\mathcal{H C}$ for which there exist a polynomial $p_{x}(\zeta)=$ $\sum_{j=0}^{N} \alpha_{j} \xi^{j}$ and a vector $z$ in $\mathcal{H}^{\prime}$ such that $p_{x}(T) x=R z, 1 / N \leqslant \sum_{j=0}^{N}\left|\alpha_{j}\right| \leqslant N$, and $\|z\| \leqslant N$. It is easy to see that each $s_{N}$ is closed, and thus $\left\{s_{N}\right\}$ is an increasing sequence of closed sets such that $\bigcup_{N=1}^{\infty} s_{N}=\mathfrak{H}$. Therefore, by the Baire category theorem, if $N_{0}$ is sufficiently large, then $s_{N_{0}}$ contains some ball

$$
B=\left\{x \in \mathcal{H}:\left\|x-x_{0}\right\|<\epsilon\right\} .
$$

Since every $x$ in $\mathcal{H C}$ can be written as $x=\beta\left(x^{\prime}-x_{0}\right)$ for some $x^{\prime}$ in $B$ and some scalar $\beta$, and since $\mathfrak{T}$ is invariant for $T$, we have $p_{x^{\prime}}(T) p_{x_{0}}(T) x \in \mathfrak{T}$. It follows that for each $x$ in $\mathcal{H C}$, there exists a nonzero polynomial $p_{x}$ of degree at most $2 N_{0}$ such that $p_{x}(T) x \in \mathfrak{N}$. The lemma now follows from Lemma 3.6 applied to the operator $T_{q}$ defined on the quotient space $\mathcal{H} / \mathfrak{N}$ by $T_{q}(x+\mathfrak{N})=T x+\mathfrak{N}$.

COROLLARY 3.8. If $T$ belongs to $\mathcal{L}(\mathcal{H C})$ and has property $(\mathrm{B})$, then for every contraction $T^{\prime}$ in $\mathscr{L}\left(\mathcal{H}^{\prime}\right)$ and every operator $X$ in $\mathscr{L}\left(\mathcal{H}^{\prime}, \mathcal{H C}\right)$ with dense range such that $T X=X T^{\prime}$, there exist a nonzero polynomial $p$ and an operator $Z$ in $\mathscr{L}\left(\mathcal{H C}, \mathcal{H C}^{\prime}\right)$ such that $p(T)=X Z$. Furthermore, the equation $p(T)=X Z$ implies $p\left(T^{\prime}\right)=Z X$ whenever $X$ is one-to-one.

Proof. Suppose $T^{\prime}$ and $X$ are as in the statement of the corollary. It follows from Definition 3.5 and Lemma 3.7 that there exists a nonzero polynomial $p$ such that $p(T) \mathfrak{H C} \subset X \mathfrak{H C}^{\prime}$. Furthermore one knows from the range inclusion theorem that there exists an operator $Z$ in $\mathscr{L}\left(\mathcal{H}, \mathcal{H}^{\prime}\right)$ satisfying $p(T)=X Z$. Suppose now that $X$ is one-to-one. Then we have $x\left(p\left(T^{\prime}\right)-Z X\right)=p(T) X-X Z X=p(T) X-p(T) X=0$, and the corollary follows at once from the injectivity of $X$.

Suppose now that $T$ is a strict contraction in $\mathscr{L}(\mathcal{H C})$. We define a large family of operators $X_{\alpha}$ each of which has dense range and intertwines $T$ and $S^{(\infty)}$. To this end we choose a sequence $\left\{h_{j}\right\}_{j=0}^{\infty}$ dense in the unit ball of $\mathcal{H}$, and for every sequence $\alpha=\left\{\alpha_{j}\right\}_{j=0}^{\infty}$ in $\left(l_{1}\right)$ we define $X_{\alpha}: \oplus_{j=0}^{\infty} H^{2} \rightarrow \mathcal{H C}$ by 


$$
X_{\alpha}\left(\bigoplus_{j=0}^{\infty} \phi_{j}\right)=\sum_{j=0}^{\infty} \alpha_{j} \phi_{j}(T) h_{j}=\sum_{j=0}^{\infty} \alpha_{j} X_{h_{j}}\left(\phi_{j}\right)
$$

in the notation of Lemma 2.1. It follows easily from that lemma that for each $\alpha$ in $\left(l_{1}\right), X_{\alpha}$ is compact and satisfies $\left\|X_{\alpha}\right\| \leqslant C\|\alpha\|_{1}$. Furthermore, a calculation shows that for each $\alpha, X_{\alpha} S^{(\infty)}=T X_{\alpha}$. Moreover, by taking $\left(\oplus_{j=0}^{\infty} \phi_{j}\right)$ successively to be 1 in the $n$th component and zeros elsewhere, $n=0,1,2, \ldots$, one sees that if $\sigma$ is defined by $\sigma=\left\{\left\{\alpha_{j}\right\} \in\left(l_{1}\right): \alpha_{j} \neq 0\right.$ for $\left.j=0,1,2, \ldots\right\}$, then whenever $\alpha \in \sigma, X_{\alpha}$ has dense range.

We are now ready for the "coup de grâce".

PROPOSITION 3.9. Every strict contraction $T$ in $\mathscr{L}(\mathcal{H C})$ having property (B) is an algebraic operator.

Proof. For each positive integer $N$, let us denote by $\sigma_{N}$ the set of all those $\alpha$ in $\left(l_{1}\right)$ for which there exist a polynomial $p_{\alpha}(\zeta)=\sum_{j=0}^{N} \gamma_{j} \zeta^{j}$ and an operator $Z_{\alpha}$ in $\mathcal{L}\left(\mathfrak{H C}, \oplus_{j=0}^{\infty} H^{2}\right)$ satisfying

$$
p_{\alpha}(T)=X_{\alpha} Z_{\alpha}, \quad 1 / N \leqslant \sum_{j=0}^{N}\left|\gamma_{j}\right| \leqslant N, \quad \text { and } \quad\left\|Z_{\alpha}\right\| \leqslant N
$$

where $X_{\alpha}$ is as in (3.5). Since the mapping $\alpha \rightarrow X_{\alpha}$ is norm continuous and the unit ball in $\mathcal{L}(\mathfrak{H C})$ is compact in the weak operator topology, it is easy to verify that each $\sigma_{N}$ is a norm-closed subset of $\left(l_{1}\right)$. Furthermore, since for each $\alpha$ in $\sigma, X_{\alpha}$ has dense range, it follows from Corollary 3.8 (with $T^{\prime}=S^{(\infty)}$ ), that $\sigma \subset \bigcup_{N=1}^{\infty} \sigma_{N}$. Moreover, since $\sigma$ is obviously a dense $G_{\delta}$ in $\left(l_{1}\right), \sigma$ is of second category in $\left(l_{1}\right)$ (cf. [5, Prob. $4 \mathrm{U}]$ ), and so, therefore, is $\bigcup_{N=1}^{\infty} \sigma_{N}$. It results that there exist a positive integer $N_{0}$ and a ball $B=\left\{\alpha \in\left(l_{1}\right):\left\|\alpha-\alpha_{0}\right\|<\epsilon\right\}$ such that $B \subset \sigma_{N_{0}}$. Since the subset of $\left(l_{1}\right)$ consisting of sequences $\alpha$ with only finitely many nonzero components is dense, there must exist a sequence $\alpha^{\prime}=\left(\alpha_{j}^{\prime}\right)_{j=0}^{\infty}$ in $B$ such that $\alpha_{j}^{\prime}=0$ for $j \geqslant J$. For this $\alpha^{\prime}$, we have, of course, $p_{\alpha^{\prime}}(T)=X_{\alpha^{\prime}} Z_{\alpha^{\prime}}$ and $T X_{\alpha^{\prime}}=X_{\alpha^{\prime}} S^{(\infty)}$. Let us write now $\mathfrak{N}=\left(\operatorname{ker} X_{\alpha^{\prime}}\right)^{\perp}$ and note that since $\alpha_{j}^{\prime}=0$ for $j \geqslant J$, we have

$$
\Re \subset \bigoplus_{j=0}^{J-1} H^{2} \quad \text { and } \quad X_{\alpha^{\prime}}=X_{\alpha^{\prime}} P_{\Re}
$$

Thus if we define $X^{\prime}$ in $\mathscr{L}(\mathfrak{N}, \mathfrak{H}), Z^{\prime}$ in $\mathscr{L}(\mathfrak{H}, \mathscr{N})$ and $T^{\prime}$ in $\mathscr{L}(\mathfrak{X})$ by

$$
X^{\prime}=X_{\alpha^{\prime}} \mid \Re, \quad Z^{\prime}=P_{\Re} Z_{\alpha^{\prime}}, \quad \text { and } \quad T^{\prime}=P_{\Re} S^{(\infty)} \mid \Re \quad\left(=P_{\Re} S^{(J)} \mid \Re\right),
$$

then it follows from (3.7) that

$$
\begin{aligned}
T X^{\prime} & =T\left(X_{\alpha^{\prime}} \mid \mathfrak{N}\right)=\left(T X_{\alpha^{\prime}}\right)\left|\mathfrak{N}=\left(X_{\alpha^{\prime}} S^{(\infty)}\right)\right| \mathfrak{N} \\
& =\left(X_{\alpha^{\prime}} P_{\Re} S^{(\infty)}\right) \mid \Re=\left(X_{\alpha^{\prime}} \mid \mathfrak{N}\right)\left(P_{\Re} S^{(J)} \mid \mathfrak{N}\right)=X^{\prime} T^{\prime}
\end{aligned}
$$

and from (3.6) and (3.7) that

$$
P_{\alpha^{\prime}}(T)=X_{\alpha^{\prime}} P_{\Re} Z_{\alpha^{\prime}}=X^{\prime} Z^{\prime} .
$$

But $X^{\prime}$ is one-to-one (as well as compact), and thus by Corollary 3.8, $p_{\alpha^{\prime}}\left(T^{\prime}\right)=Z^{\prime} X^{\prime}$, which implies that $T^{\prime}$ is polynomially compact. Observe next that $T^{\prime}$ is covered by 
Remark 2.4, and therefore by Corollary 2.3 there exist disjoint subspaces $\Re_{0}$ and $\Re_{1}$ of $\mathscr{N}$ such that $\mathfrak{K}_{0}+\mathscr{N}_{1}=\mathscr{N}, \operatorname{dim} \mathfrak{N}_{0}<\aleph_{0}, T^{\prime} \mathfrak{N}_{1} \subset \mathfrak{N}_{1}$, and

$$
\sigma\left(T^{\prime} \mid \Re_{1}\right) \cap\{\zeta:|\zeta| \leqslant\|T\|\}=\varnothing .
$$

Moreover, (3.9) implies that $T\left(X^{\prime} \mid \Re_{1}\right)=\left(X^{\prime} \mid \Re_{1}\right)\left(T^{\prime} \mid \Re_{1}\right)$. Thus, by a theorem of Lumer and Rosenblum [9], it follows that $X^{\prime} \mid \mathfrak{N}_{1}=0$, and hence that $\mathfrak{N}_{1}=0$, since $X^{\prime}$ is one-to-one. We conclude that $\mathfrak{N}=\mathfrak{N}_{0}$ is finite dimensional, and thus from (3.8) and (3.10) that $p_{\alpha^{\prime}}(T)$ has finite rank. If $q$ is any nonzero polynomial such that $q\left(p_{\alpha^{\prime}}(T)\right)=0$, then $\left(q \circ p_{\alpha^{\prime}}\right)(T)=0$, and the proof is complete.

The following corollary follows immediately from the remark after Definition 3.5.

COROLLARY 3.10. Every strict contraction $T$ in $\mathscr{L}(\mathcal{H C})$ that has property (A) is algebraic.

We are now ready for the main result of this section.

THEOREM 3.11. If $T$ is a nonalgebraic operator in $\mathscr{L}(\mathcal{H C})$ and $\|T\|<\alpha$, then $\alpha S^{(\infty)} \prec T$.

Proof. We may write $\alpha=\gamma / \beta$ where $\beta<1 /\|T\|$ and $1<\gamma$. Then $\beta T$ is a strict contraction and by Corollary 3.10, $T$ cannot have property (A). Thus, by Proposition 3.4, we have $\gamma S^{(\infty)} \prec \beta T$, and this is equivalent to $\alpha S^{(\infty)} \prec T$.

We remark that it is an easy consequence of a theorem of Rota [11] that the conclusion of Theorem 3.11 holds under the weaker assumption that $\alpha$ is any number larger than the spectral radius of $T$.

We also remark that a perusal of the proofs of the results in this section shows that the fact that the operator $T$ acts on a Hilbert space plays no essential role, and therefore we have established the following more general result.

THEOREM 3.12. If $T$ is a nonalgebraic (bounded, linear) operator acting on a separable complex Banach space and $\|T\|<\alpha$, then $\alpha S^{(\infty)}<T$.

Let us observe that the conclusion of Theorem 3.11 is generally false for $\alpha=\|T\|$. Indeed, if $T$ is a completely nonunitary contraction and $S^{(\infty)} \prec T$, then $u\left(S^{(\infty)}\right) \prec$ $u(T)$ for every $u$ in $H^{\infty}$, and therefore $u(T) \neq 0$ whenever $u \neq 0$. Thus, if $T$ is a nonalgebraic $C_{0}$-contraction, then one knows that $\|T\|=1$ and the above argument shows that $S^{(\infty)}$ is not a quasiaffine transform of $T$.

REMARK 3.13. It is known that $S \prec S^{*}$. Indeed, if $T$ is any strict contraction such that both $T$ and $T^{*}$ have cyclic vectors, then it follows from [16] that $S \prec T \prec S^{*}$. Furthermore, if $X$ is any quasiaffinity satisfying $X S=S^{*} X$, and $\mathfrak{T}$ is any nonzero invariant subspace for $S$, then $X \mathfrak{T}$ is dense in $H^{2}$. (For, if $(X \mathfrak{T})^{-} \neq H^{2}$, then $S^{*}(X \mathfrak{T})^{-} \subset(X \mathfrak{T})^{-}$and $S^{*} \mid(X \mathfrak{T})^{-}$is a $C_{0^{-}}$-operator satisfying $S \mid \mathfrak{T} \prec$ $S^{*} \mid(X \mathfrak{N})^{-}$, contradicting the fact that $S \mid \mathfrak{T}$ is unitarily equivalent to $S$, which is not a $C_{0}$-operator.) Observe next that Theorem 3.11 implies that

$$
S^{(\infty)} \prec S / 2 \prec S^{*} / 2 \prec S^{(\infty) *},
$$


and if $Y$ is the quasiaffinity satisfying $Y S^{(\infty)}=S^{(\infty) *} Y$ obtained by composing the three quasiaffinities given by (3.11), then it follows easily from the above result about $X$ that if $\Re$ is any nonzero invariant subspace for $S^{(\infty)}$, then $Y \Re$ is dense in $\mathfrak{H}^{(\infty)}$.

4. Normal plus compact quasiaffine transforms. In this section we will prove that every operator $T$ in $\mathscr{L}(\mathfrak{H})$ has a quasiaffine transform of the form $N+K$, where $N$ is normal and $K$ is a compact operator of arbitrarily small norm. We will also show that the relation $N+K \prec T$ implies $N+K \sim T$ if $T$ is algebraic but not in general. Finally, we will investigate cases in which an operator $T$ has either a normal or a compact quasiaffine transform. The program begins with two lemmas.

LEMMA 4.1. Let $W_{\omega}$ be a unilateral weighted shift in $\mathcal{L}\left(H^{2}\right)$ with weight sequence $\omega=\left\{w_{j}\right\}_{j=0}^{\infty}$, where $w_{j}>0$ for all $j$. Then $W_{\omega} \prec S$ whenever

$$
\inf \left\{w_{0} w_{1} \ldots w_{n}: n \geqslant 0\right\}>0 .
$$

Proof. If (4.1) holds, there exists an operator $X$ in $\mathcal{L}\left(H^{2}\right)$ such that $X e_{0}=e_{0}$ and $X e_{n}=\left(1 / w_{0} w_{1} \ldots w_{n-1}\right) e_{n}, n=1,2, \ldots$ It is obvious that $X$ is a quasiaffinity, and an easy calculation shows that $X W_{\omega}=S X$, from which the result follows.

LEMMA 4.2. Let $\left\{\epsilon_{j}\right\}_{j=0}^{\infty}$ be a decreasing sequence of positive numbers converging to zero. Then there exists a sequence $\left\{k_{j}\right\}_{j=0}^{\infty}$ of positive integers such that the weighted shift $W_{\omega}$ with weight sequence $\omega$ given by

$$
\omega\left(\epsilon_{0}, k_{0}\right), \epsilon_{0}, \omega\left(\epsilon_{1}, k_{1}\right), \epsilon_{1}, \ldots, \omega\left(\epsilon_{j}, k_{j}\right), \epsilon_{j}, \ldots,
$$

where the finite sequences $\omega\left(\epsilon_{i}, k_{i}\right)$ are as in (2.3), is a quasiaffine transform of $S$ and satisfies $\left\|W_{\omega}\right\| \leqslant 1+\epsilon_{0}$. Moreover, $W_{\omega}$ can be written as $W_{\omega}=N+K$, where $N$ is normal, $\|N\| \leqslant 1+2 \epsilon_{0}+200 \epsilon_{0}^{1 / 2}, K$ is compact, and $\|K\| \leqslant \epsilon_{0}+200 \epsilon_{0}^{1 / 2}$.

Proof. For each nonnegative integer $j$, let $n_{j}$ be the smallest positive integer such that $n_{j} \epsilon_{j}>1$, and observe from (2.3) that if the positive integer $k_{j}$ is chosen so large that

$$
\left(n_{j} \epsilon_{j}\right)^{k_{j}}\left(n_{j}-1\right) ! \epsilon_{j}^{n_{j}}\left(n_{j+1}-1\right) ! \epsilon_{j+1}^{n_{j+1}-1}>1,
$$

then the weight sequence $\omega$ certainly satisfies (4.1), so we have $W_{\omega} \prec S$ by Lemma 4.1 and $\left\|W_{\omega}\right\| \leqslant 1+\epsilon_{0}$ by inspection. Moreover it is obvious that we may write $W_{\omega}$ as $W_{\omega}=W_{\omega^{\prime}}+K^{\prime}$ where $\omega^{\prime}$ is the weight sequence

$$
\omega\left(\epsilon_{0}, k_{0}\right), 0, \omega\left(\epsilon_{1}, k_{1}\right), 0, \ldots, \omega\left(\epsilon_{j}, k_{j}\right), 0, \ldots,
$$

$K^{\prime}$ is compact, and $\left\|K^{\prime}\right\| \leqslant \epsilon_{0}$. Furthermore it is clear that $W_{\omega^{\prime}}$ is unitarily equivalent to $\bigoplus_{j=0}^{\infty} T_{\omega\left(\epsilon_{j}, k_{j}\right)}$, where the $T_{\omega\left(\epsilon_{j}, k_{j}\right)}$ are as in (2.2). Thus, by Theorem 2.5, we may write $T_{\omega\left(\epsilon_{j}, k_{j}\right)}=N_{j}+K_{j}$ where $N_{j}$ is normal and $\left\|K_{j}\right\| \leqslant 200 \epsilon_{j}^{1 / 2}$. Since

$$
\bigoplus_{j=0}^{\infty} T_{\omega\left(\epsilon_{j}, k_{j}\right)}=\left(\bigoplus_{j=0}^{\infty} N_{j}\right)+\left(\bigoplus_{j=0}^{\infty} K_{j}\right) \text {, }
$$

the result follows by setting $N=\oplus_{j=0}^{\infty} N_{j}, K=K^{\prime}+\oplus_{j=0}^{\infty} K_{j}$, and noting that since $\epsilon_{j} \rightarrow 0, \oplus_{j=0}^{\infty} K_{j}$ is compact and we have 


$$
\begin{aligned}
& \|K\| \leqslant \epsilon_{0}+200 \epsilon_{0}^{1 / 2}, \\
& \|N\|=\left\|W_{\omega}-K\right\| \leqslant\left\|W_{\omega}\right\|+\|K\| \leqslant 1+2 \epsilon_{0}+200 \epsilon_{0}^{1 / 2} .
\end{aligned}
$$

We can now prove the universality of the existence of normal plus compact quasiaffine transforms.

THEOREM 4.3. Suppose $T$ belongs to $\mathscr{L}(\mathcal{H})$ and $\epsilon$ is any positive number. Then there exist $N$ and $K$ in $\mathcal{L}(\mathcal{H C})$ such that $N$ is normal, $K$ is compact, $N+K \prec T$, and $\|K\| \leqslant \epsilon$. If $T$ is algebraic, we also have $T \prec N+K$, so $T$ and $N+K$ are quasisimilar.

Proof. We suppose first that $T$ is not algebraic. In this case it follows easily from Theorem 3.11 that it suffices to prove the theorem when $T=S^{(\infty)}$. Let $\left\{\epsilon_{j}\right\}_{j=0}^{\infty}$ be a decreasing sequence of positive numbers converging to zero and satisfying

$$
\epsilon_{0}+200 \epsilon_{0}^{1 / 2} \leqslant \epsilon, \quad 1+2 \epsilon_{0}+200 \epsilon_{0}^{1 / 2} \leqslant 2 .
$$

Then, by Lemma 4.2, for each positive integer $j$, there exist a normal operator $N_{j}$ and a compact operator $K_{j}$ such that $N_{j}+K_{j}<S$ and $\left\|N_{j}\right\| \leqslant 2,\left\|K_{j}\right\| \leqslant \epsilon_{j}+200 \epsilon_{j}^{1 / 2}$. We set $N=\oplus_{j=0}^{\infty} N_{j}, K=\oplus_{j=0}^{\infty} K_{j}$, and observe that $N$ is normal, $K$ is compact, $\|K\| \leqslant \epsilon$, and $N+K \prec S^{(\infty)}$. Thus the proof is complete in the case when $T$ is not algebraic.

That an algebraic operator $T$ in $\mathscr{L}(\mathcal{H C})$ is quasisimilar to an operator of the form $N+K$, where $N$ is normal, $K$ is compact, and $\|K\|$ is arbitrarily small, is well known. For completeness, we sketch an argument. It can easily be seen that $T$ is similar to an operator of the form $\oplus_{j=1}^{n}\left(\lambda_{j}+N_{j}\right)$ where each $N_{j}$ is a nilpotent operator. (This follows, for example, from the Riesz-Dunford functional calculus or from a theorem of Halmos [8] and the theorem, used above several times, of Lumer and Rosenblum [9].) Furthermore, one knows from [7, Theorem 4] that every nilpotent operator is quasisimilar to a compact operator of arbitrarily small norm, and the result follows easily by forming the appropriate direct sums.

The last statement of the theorem follows from the fact, mentioned in the introduction, that if $T^{\prime}<T$ and $T$ is algebraic, then $T^{\prime}$ is also algebraic and $T^{\prime} \sim T$ (cf. [2], [19]).

The following proposition is useful in showing that we cannot expect all operators to have normal quasiaffine transforms. We denote the point spectrum of an operator $T$ in $\mathcal{L}(\mathcal{H C})$ by $\sigma_{p}(T)$ and the set of complex conjugates of a subset of $\Lambda$ of $\mathbf{C}$ by $\Lambda^{*}$.

PROPOSITION 4.4. Suppose that $N$ and $T$ are operators in $\mathscr{L}(\mathcal{H C})$ such that $N$ is normal and $N<T$. Then $\sigma(N) \subset \sigma(T)$ and $\left[\sigma_{p}\left(T^{*}\right)\right]^{*} \subset \sigma_{p}(N) \subset \sigma_{p}(T)$.

Proof. The first inclusion follows from Corollary 2.12 of [6]. For completeness, we sketch an argument. Let $X$ be a quasiaffinity such that $X N=T X$, and suppose first that $\sigma(N) \backslash \sigma(T) \neq \varnothing$. Then there exists an open set $\mathcal{U}$ such that $\mathcal{U}^{-} \subset \sigma(N) \backslash \sigma(T)$ and $E=E\left(\mathcal{U}^{-}\right) \neq 0$, where $E(\cdot)$ is the spectral measure associated with $N$. Thus $(X E)(E N)=X E N=X N E=T(X E)$, and since $\sigma(E N) \subset \mathcal{U}^{-} \subset \mathbf{C} \backslash \sigma(T)$, the theorem of Lumer and Rosenblum [9] implies that $X E=0$, contradicting the fact that $X$ is a quasiaffinity. Hence $\sigma(N) \subset \sigma(T)$. That $\sigma_{p}\left(T^{*}\right) \subset \sigma_{p}\left(N^{*}\right)$ and $\sigma_{p}(N) \subset \sigma_{p}(T)$ follow from the obvious general facts that if $T_{1} \prec T_{2}$, then $T_{2}^{*} \prec T_{1}^{*}$ and $\sigma_{p}\left(T_{1}\right) \subset \sigma_{p}\left(T_{2}\right)$. Since $\sigma_{p}\left(N^{*}\right)=\left[\sigma_{p}(N)\right]^{*}$, the desired inclusion relations result. 
REMARK 4.5. Proposition 4.4 shows clearly that one cannot hope to show that every operator in $\mathscr{L}(\mathcal{H C})$ has a normal quasiaffine transform. For example, if $\sigma_{p}(T)=\varnothing$ but $\sigma_{p}\left(T^{*}\right) \neq \varnothing$, then $N \prec T$ is impossible for every normal operator $N$. (In particular, the operator $S$ has no normal quasiaffine transform.) Furthermore, no nonzero nilpotent operator can have a normal or even a cohyponormal quasiaffine transform as is shown by Theorem 3.6 of [6].

REMARK 4.6. The operator $W_{\omega}$ considered in Lemma 4.2 is interesting in itself. It has the form $W_{\omega}=N+K$ with $N$ normal and $K$ compact, but for no normal operator $N^{\prime}$ do we have $N^{\prime} \prec W_{\omega}$. Indeed, $N^{\prime} \prec W_{\omega}$ would imply (by Lemma 4.2) that $N^{\prime} \prec S$, contradicting Remark 4.5. It is also easy to see that $\left\|\pi\left(W_{\omega}\right)\right\| \leqslant 1$. (This follows from the fact that since $\epsilon_{j} \rightarrow 0$ and no weight in the sequence $\omega\left(\epsilon_{j}, k_{j}\right)$ exceeds $1+\epsilon_{j}$, $W_{\omega}$ can be perturbed by a compact weighted shift $K^{\prime}$ such that the weighted shift $W_{\omega}-K^{\prime}$ satisfies $\left\|W_{\omega}-K^{\prime}\right\|=1$.) Thus $\sigma_{e}\left(W_{\omega}\right) \subset D^{-}=\{\zeta:|\zeta| \leqslant 1\}$. On the other hand, the relation $W_{\omega} \prec S$ obviously implies $\sigma_{p}\left(W_{\omega}\right) \subset \sigma_{p}(S)=\varnothing$ as well as $D=$ $\sigma_{p}\left(S^{*}\right) \subset \sigma_{p}\left(W_{\omega}^{*}\right)$. Since $W_{\omega}=N+K$, any hole in $\sigma_{e}\left(W_{\omega}\right)$ must have associated Fredholm index 0 , but since $\sigma_{p}\left(W_{\omega}\right)=\varnothing$, no such hole can exist. Thus we see that $\sigma_{e}\left(W_{\omega}\right)=D^{-}$and that $W_{\omega}=N+K$ has the curious property that its point spectrum is empty but the point spectrum of its adjoint $(N+K)^{*}$ contains $D$.

REMARK 4.7. It is known (cf. [7]) that there exist quasinilpotent operators in $\mathscr{L}(\mathcal{H C})$ that do not commute with any nonzero compact operator. For such a $T$ we cannot replace the relation $N+K \prec T$ of Theorem 4.3 by quasisimilarity, as is shown by the following result.

THEOREM 4.8. If $T$ is an operator in $\mathcal{L}(\mathcal{H C})$ such that $\sigma_{e}(T)=\{0\}$ and $T \sim N+K$, where $N$ is normal in $\mathscr{L}(\mathcal{H C})$ and $K$ is compact, then $T$ commutes with a nonzero compact operator.

Proof. Let $X$ and $Y$ be two quasiaffinities in $\mathscr{L}(\mathcal{H C})$ such that

$$
T X=X(N+K) \quad \text { and } \quad Y T=(N+K) Y .
$$

If $T=0$, the theorem is trivial, so we now suppose that $T \neq 0$. Then clearly $X(N+K) Y$ is nonzero and commutes with $T$, so it is enough to show that this operator is compact. Furthermore, this can be done by showing that $X N$ is compact, and since $\left\|N-E_{n} N E_{n}\right\| \rightarrow 0$, where $E_{n}$ is the spectral projection of $N$ corresponding to the set $\Lambda_{n}=\{\zeta: 1 / n \leqslant|\zeta|\}$, it suffices to show that for each positive integer $n$, $X E_{n} N E_{n}$ is compact. Note that in the Calkin algebra, we have, by (4.2),

$$
\pi(T) \pi\left(X E_{n}\right)=\pi\left(X E_{n}\right) \pi\left(E_{n} N E_{n}\right)=\pi\left(X E_{n}\right) \pi\left(E_{n} N E_{n}+1-E_{n}\right),
$$

and since $\sigma_{e}(T)=\{0\}$ and $\sigma\left(E_{n} N E_{n}+1-E_{n}\right) \subset \Lambda_{n} \cup\{1\}$, it follows from the theorem of Lumer and Rosenblum [9] (applied in the Calkin algebra) that $X E_{n}$ is compact for every $n$, thus completing the proof.

Our last result is a generalization of [7, Theorem 3] and shows that for a certain class of operators $T$ the relation $N+K \prec T$ in Theorem 4.3 can be replaced by a stronger relation. 
THEOREM 4.9. Suppose $T$ is an operator in $\mathcal{L}(\mathcal{H C})$ such that $\sigma_{e}(T)=\{0\}$. Then there exist compact operators $K^{\prime}$ and $K^{\prime \prime}$ in $\mathcal{L}\left(\mathcal{F C}^{\prime}\right)$ such that $K^{\prime}<T<K^{\prime \prime}$.

Proof. The relation $K^{\prime} \prec T$ is equivalent to $T^{*} \prec\left(K^{\prime}\right)^{*}$, so it suffices to establish the existence of $K^{\prime \prime}$. Furthermore, to do this it is enough to prove the existence of a compact operator $K_{1}$ in some $\mathscr{L}\left(\mathcal{H C}^{\prime \prime}\right)$ and an injective $Z$ in $\mathscr{L}\left(\mathcal{H C}, \mathcal{H C}^{\prime \prime}\right)$ such that

$$
K_{1} Z=Z T \text {. }
$$

Indeed, in this case we can set $\mathcal{F}^{\prime}=(Z \mathcal{H})^{-}$and $K^{\prime \prime}=K_{1} \mid \mathcal{H}^{\prime}$. Thus we will establish (4.3). By Proposition 2.7 we can write $T=T^{\prime}+K_{2}$ where $T^{\prime}$ is quasinilpotent and $K_{2}$ is compact. Furthermore, by Theorem 2.6 there exist a quasinilpotent, compact $K_{3}$ in $\mathscr{L}(\mathcal{H})$ and an $X$ in $\mathscr{L}\left(\mathcal{H}, \mathcal{H}^{(\infty)}\right)$ that is bounded below such that $K_{3}^{(\infty)} X=$ $X T^{\prime}$. Let us set $\mathcal{H}_{1}=X \mathcal{H C}$ and define a compact operator $K_{4}$ in $\mathcal{L}^{\left(\mathcal{H}^{(\infty)}\right)}$ by $K_{4}=X K_{2} X^{-1} P_{\mathfrak{K}_{1}}$, where $P_{\mathfrak{K C}_{1}}$ is the (orthogonal) projection of $\mathcal{F C}^{(\infty)}$ on $\mathfrak{H C}_{1}$ and $X^{-1}: \mathcal{H C}_{1} \rightarrow \mathcal{H C}$ is the "inverse" of $X$. Then, obviously, we have $K_{4} X=X K_{2}$, and hence

$$
\left(K_{3}^{(\infty)}+K_{4}\right) X=X T \text {. }
$$

If $K_{3}^{(\infty)}$ were compact, then (4.3) would be established since $X$ is injective. Since $K_{3}^{(\infty)}$ is not compact, we must tinker further with (4.4). Let $Y$ be any quasiaffinity in $\mathcal{L}\left(\mathcal{H C}^{(\infty)}\right)$, and write $Y^{-1}$ for the densely defined, possibly unbounded, set theoretic inverse of $Y$. Then, formally, we may write

$$
Y\left(K_{3}^{(\infty)}+K_{4}\right) Y^{-1} Y X=Y X T,
$$

and since $Y X$ is injective, it suffices to construct some quasiaffinity $Y$ in $\mathscr{L}\left(\mathcal{H}^{(\infty)}\right)$ such that $Y K_{3}^{(\infty)} Y^{-1}$ and $Y K_{4} Y^{-1}$ are bounded (on the range of $Y$ ) and such that the extensions of these densely defined operators to $\mathcal{J C}^{(\infty)}$ are both compact.

Since $K_{3}$ is quasinilpotent, by a theorem of Rota [11], there exists a sequence $\left\{Q_{j}\right\}_{j=0}^{\infty}$ of invertible operators in $\mathscr{L}(\mathcal{H C})$ such that $Q_{0}=1$ and for each $j \geqslant 1$, $\left\|Q_{j}\right\|=1,\left\|Q_{j} K_{3} Q_{j}^{-1}\right\| \leqslant 1 / 2^{j}$. Furthermore, if we denote by $P_{n}$ the projection of $\mathfrak{H C}^{(\infty)}=\bigoplus_{j=0}^{\infty} \mathfrak{H C}$ onto $\bigoplus_{j=n}^{\infty} \mathfrak{H}, n=1,2, \ldots$, then $\left\|K_{4} P_{n}\right\| \rightarrow 0$ (cf. [10, Corollary 4.4]), and thus we may choose an increasing sequence $\left\{n_{j}\right\}_{j=0}^{\infty}$ of natural numbers such that $n_{0}=0$ and

$$
\left\|K_{4} P_{n_{j}}\right\|\left\|Q_{j}^{-1}\right\|<1 / 2^{j}, \quad j=1,2, \ldots .
$$

For each nonnegative integer $j$, we define $Y_{j}=Q_{k}$ where $k$ satisfies $n_{k} \leqslant j<n_{k+1}$, and we set $Y=\bigoplus_{j=0}^{\infty} Y_{j}$. Then $Y K_{3}^{(\infty)} Y^{-1}$ can be written as $\bigoplus_{j=0}^{\infty} Y_{j} K_{3} Y_{j}^{-1}$ and this operator can be extended to a compact operator in $\mathscr{L}\left(\mathcal{H}^{(\infty)}\right)$ since

$$
\left\|Y_{j} K_{3} Y_{j}^{-1}\right\| \rightarrow 0 \text {. }
$$

Finally, the operator $Y K_{4} Y^{-1}$ can be written as

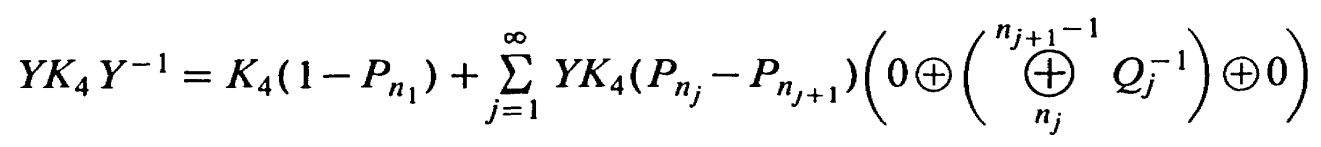

and this operator also extends to a compact operator on $\mathcal{H}^{(\infty)}$ since for every positive integer $j$, 


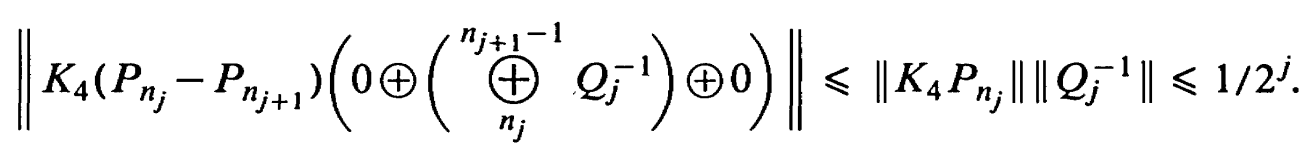

Thus the theorem is proved.

\section{REFERENCES}

1. C. Apostol, Quasiaffine transforms of quasinilpotent operators. Rev. Roumaine Math. Pures Appl. 21 (1976), 813-816.

2. C. Apostol, R. G. Douglas and C. Foiaş, Quasisimilar models for nilpotent operators. Trans. Amer. Math. Soc. 224 (1976), 407-415.

3. C. Apostol, C. Pearcy and N. Salinas, Spectra of compact perturbations of operators. Indiana Univ. Math. J. 26 (1977), no. 2, 345-350.

4. I. D. Berg, On approximation of normal operators by weighted shifts. Michigan Math. J. 21 (1975), 377-383.

5. A. Brown and C. Pearcy, Introduction to operator theory, I. Elements of functional analysis, Springer, New York, 1977.

6. L. A. Fialkow, A note on quasisimilarity of operators. Acta Sci. Math. (Szeged) 39 (1977), 67-85.

7. C. Foiaş and C. Pearcy, A model for quasinilpotent operators. Michigan Math. J. 21 (1975), 399-404.

8. P. R. Halmos, Capacity in Banach algebras. Indiana Univ. Math. J. 20 (1971), 855-863.

9. G. Lumer and M. Rosenblum, Linear operator equations. Proc. Amer. Math. Soc. 10 (1959), 32-41.

10. C. Pearcy, Some recent developments in operator theory. CBMS Regional Conference Series, No. 36, Amer. Math. Soc., Providence, 1978.

11. G. C. Rota, On models for linear operators. Comm. Pure Appl. Math. 13 (1960), 468-472.

12. B. Sz.-Nagy and C. Foiaş, Vecteurs cycliques et quasi-affinités. Studia Math. 31 (1968), $35-42$.

13. - Harmonic analysis of operators on Hilbert space, North-Holland, Amsterdam, 1970.

14. - Modèles de Jordan pour une classe d'opérateurs de l'espace de Hilbert. Acta Sci. Math. (Szeged) 31 (1970), 91-115.

15. — Compléments à l'étude des opérateurs de classe $C_{0}$. Acta Sci. Math. (Szeged) 31 (1970), 287-296.

16. - Injections of shifts into strict contractions. Linear operators and approximation, II (Proc. Conf., Oberwolfach Math. Res. Inst., Oberwolfach, 1974), pp. 29-37, Internat. Ser. Numer. Math., Vol. 25, Birkhäuser, Basel, 1974.

17. - On injections intertwining contractions of class $C_{0}$. Acta Sci. Math. (Szeged) 40 (1978), 163-167.

18. T. West, The decomposition of Riesz operators. Proc. London Math. Soc. 16 (1966), 737-752.

19. L. R. Williams, $A$ quasisimilarity model for algebraic operators. Acta Sci. Math. (Szeged) 40 (1978), no. 1-2, 185-188.
C. Apostol
Department of Math.
INCREST
Bucharest, Romania

\author{
H. Bercovici \& C. Pearcy \\ Department of Mathematics \\ University of Michigan \\ Ann Arbor, MI 48109
}

\author{
C. Foiaş \\ Department of Mathematics \\ Indiana University \\ Bloomington, IN 47401
}

